\title{
Introduction to the Special Issue on Physician Mental Health
}

\author{
Pamela Wible \\ Family Physician, Founder Ideal Medical Care, Eugene, OR, USA
}

Amid tragedy, a doctor's most heroic act may be to listen - to be a sacred witness to the suffering of patients. Yet where do heroic doctors go with their pain? Who listens to the suffering of physicians? The guilt of not being able to do more for patients. The burden of delivering devastating news to families. The work-related anxiety and depression due to unrealistic expectations of perfectionism. Few venues exist for medical students and physicians to share their uncensored lived experiences. Welcome to a special issue of Qualitative Research in Medicine and Healthcare dedicated to physician mental health and the unexplored lives of our wounded healers.

Doctor suicide has been a well-hidden occupational hazard of the medical profession for more than a century. Though students enter medicine with their mental health on par with or better than their peers, they are up to three times more likely to kill themselves according to the American Medical Student Association. In some residency programs $75 \%$ of interns meet criteria for major depression. We know that suicide risk increases with untreated mental illness. Physicians who die by suicide are less likely to be receiving mental health care compared with non-physician suicides. In fact, doctors are more likely to self-medicate for anxiety, depression, and suicidality - with tragic outcomes. Why?

Here physicians share their personal journeys with anxiety, depression, PTSD, and suicide amid life-and- death decisions for patients - and themselves. Some doctors have prevailed. Others have been injured by the very agencies claiming to support them. A few have lost careers due to seeking help for occupationally induced mental health wounds.

So how do we harness health in a system that undermines the mental health of our healers? Well - meaning medical institutions offer physician wellness activities with ice cream, yoga, and chamomile tea, yet these programs have been less effective at addressing the vicarious trauma doctors sustain from helping victims of child abuse, domestic violence, and social injustice. To create a culture of wellness in medicine, simple foundational solutions are proposed and celebrated here such as the recent decision by the National Board of Medical Examiners to change the United States Medical Licensing Examination from a numeric grade to pass/fail and the Federation of State Medical Board's decision to remove questions from licensing applications that may violate the Americans with Disabilities Act.

Eradicating mental health stigma from within medicine allows our healers to receive the non-punitive psychological support they require - and as a result we all benefit.

May you enjoy this unique opportunity to explore physician psychology.
Correspondence: Pamela Wible, P.O. Box 5225, Eugene, OR 97405, USA.

Tel.: +1.541.345.2437.

E-mail: roxywible@comcast.net

Received for publication: 29 October 2019.

Accepted for publication: 12 November 2019.

This work is licensed under a Creative Commons Attribution NonCommercial 4.0 License (CC BY-NC 4.0).

${ }^{\circ}$ Copyright: the Author(s), 2019

Licensee PAGEPress, Italy

Qualitative Research in Medicine \& Healthcare 2019; 3:98

doi:10.4081/qrmh.2019.8922 